\title{
Absence of the Effects of Vortices in the Gauge Glass
}

\author{
Toshiyuki Hamasaki and Hidetoshi Nishimori \\ Department of Physics, Tokyo Institute of Technology, Oh-okayama, Meguro-ku, \\ Tokyo 152-8551, Japan
}

\begin{abstract}
We calculate several correlation functions and distribution functions of dynamical variables for the gauge glass and the Villain model using the spin wave approximation and the gauge transformation. The results show that the spin wave approximation gives the exact solutions on the Nishimori line in the phase diagram. This implies that vortices play no role in the thermodynamic behavior of the system as long as some correlation and distribution functions are concerned. These results apply to any dimensions including the two-dimensional case.
\end{abstract}

Key words: periodicity; gauge transformation; Nishimori line

\section{Introduction}

The gauge glass, which is an extension of the $X Y$ model to the random spin system, attracts both theoretical and practical interest. This model provides good descriptions of some physical situations, for example, the $X Y$ magnet with random Dzyaloshinskii-Moriya interactions [1] and a granular superconductivity and Josephson-junction arrays with positional disorder [2][3]. Especially, the two-dimensional gauge glass has been studied actively because of the interesting relation between the Kosterlitz-Thouless (KT) transition and the effect of disorder. This relation was first discussed by Rubinstein, Shraiman and Nelson [1]. They have shown that for small amount of randomness, as the temperature is decreased, there appears first the paramagnetic phase, then a KT-like phase, and finally again a paramagnetic phase. Thus a reentrant transition appears in the gauge glass. After their work, Natterman, Scheidl, Korshunov and $\mathrm{Li}$ [4] corrected their description of the reentrant transition to the one without reentrance. These latter authors pointed out an overestimation of vortex pair density in the previous work. Furthermore, Sheidl [5] found a new ordered phase in which single vortex excitations occur in the 
reentrant phase, and Maucourt and Grempel [6] suggested from Monte Carlo simulations that there is no indication of a low-temperature reentrant phase. Today, the two-dimensional gauge glass at low temperatures is generally believed to have no reentrance [7], except for some researchers [8]. Hence, the present major interest in the two-dimensional gauge glass is the structure of the KT phase, namely, the boundary between the freezing phase of the vortexpair excitations and the non-freezing phase.

The gauge glass has also been investigated in the field of the spin glass theory because the gauge glass is an extension of the Ising spin glass with continuous spin variables [9]. Particularly, the method of gauge transformation, which was developed in the study of the spin glass, is a powerful technique for deriving analytical results for gauge glass. Ozeki and Nishimori [10] found the exact solution of the internal energy of the gauge glass under a special condition using the method of gauge transformation. This special condition relevant to the exact solution corresponds to a line in the phase diagram called the Nishimori line. Ozeki and Nishimori also showed that the phase boundary between the ordered and the disordered phases runs parallel to the temperature axis. Although they could obtain the exact solution on the Nishimori line, there exists a mysterious property associated with the line: For example, the exact solution of the internal energy on the Nishimori line has no singularity as a function of temperature although the the line runs across the phase boundary. From these facts in mind, we aim to clarify what occurs under the special condition of this line.

In this paper, we calculate some gauge invariant quantities on the Nishimori line. First, we define the gauge glass and show that the exact solution of the internal energy can be calculated under the special condition in Section 2. Next, we introduce the gauge invariant correlation functions and calculate them using the spin wave approximation, gauge transformation and the Villain model in Section 3. The gauge invariant distribution functions are introduced and calculated in Section 4. From the results of Sections 3 and 4, we discuss the physical properties of the Nishimori line in Section 5.

\section{General properties}

\subsection{Gauge glass}

The gauge glass is defined by the Hamiltonian

$$
H=-J \sum_{\langle i, j\rangle} \cos \left(\theta_{i}-\theta_{j}-A_{i j}\right),
$$


where $A_{i j}$ is the quenched random phase shift and the coupling constant $J$ is positive. The sum runs over all nearest-neighbor pairs on a lattice. Note that we do not specify the spatial dimensionality explicitly here. We assume that $A_{i j}$ is independently distributed at each bond as follows,

$$
P\left(A_{i j}\right)=\frac{1}{2 \pi I_{0}\left(K_{p}\right)} \mathrm{e}^{K_{p} \cos A_{i j}}
$$

where the magnitude of $K_{p}$ controls the tendency toward ferromagnetism; the system becomes the pure ferromagnetic $X Y$ model in the limit of large $K_{p}$. The function $I_{0}(x)$ appearing in the denominator of eqn $(2)$ is the modified Bessel function $I_{0}(x)=\int_{0}^{2 \pi} \mathrm{d} \theta \mathrm{e}^{x \cos \theta}$. Note that we express the thermal average by angular brackets $\langle\cdots\rangle$ and the average over the distribution of quenched randomness by square brackets $[\cdots]$.

\subsection{Exact solution of the internal energy}

The gauge transformation is a powerful method to calculate the exact solution of the internal energy for the gauge glass [11]. We introduce the gauge transformation to our model and derive the exact internal energy under a special condition.

The gauge transformation is defined as

$$
\theta_{i} \rightarrow \theta_{i}-\phi_{i}, \quad A_{i j} \rightarrow A_{i j}-\phi_{i}+\phi_{j}
$$

where $\phi_{i}$ is a gauge variable fixed arbitrarily at each site. The Hamiltonian (1) is invariant under this transformation. The internal energy is written explicitly as

$$
E=[\langle H\rangle]=\int \prod_{\langle i, j\rangle} \frac{\mathrm{d} A_{i j}}{2 \pi} \frac{\mathrm{e}^{K_{p} \cos A_{i j}}}{I_{0}\left(K_{p}\right)} \cdot \frac{\int \prod_{i} \frac{\mathrm{d} \theta_{i}}{2 \pi} H \mathrm{e}^{K \sum_{\langle i, j\rangle} \cos \left(\theta_{i}-\theta_{j}-A_{i j}\right)}}{\int \prod_{i} \frac{\mathrm{d} \theta_{i}}{2 \pi} \mathrm{e}^{K \sum_{\langle i, j\rangle} \cos \left(\theta_{i}-\theta_{j}-A_{i j}\right)}},
$$

where the ranges of integration over $A_{i j}$ and $\theta_{i}$ are from 0 to $2 \pi$. Here we apply the gauge transformation to eqn (4). Since the gauge transformation is just a change of running variables, the value of eqn (4) is independent of the choice of $\left\{\phi_{i}\right\}$ and therefore we may integrate eqn (4) over all gauge variables $\left\{\phi_{i}\right\}$, each from 0 to $2 \pi$, and divide each integration of $\phi_{i}$ by $2 \pi$. Using the special condition $K=K_{p}$, we obtain the exact internal energy as 


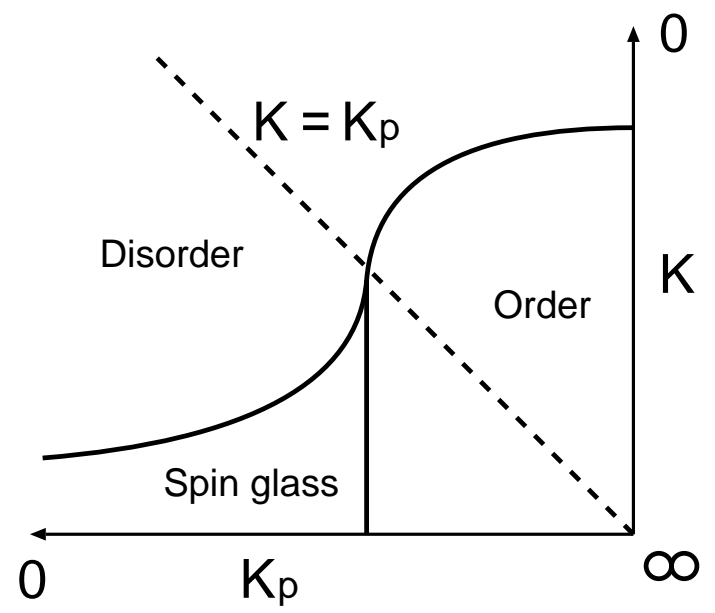

Fig. 1. The schematic phase diagram and the Nishimori line (dashed). The spin glass phase exists in three dimensions or over.

$$
E=-J N_{B} \frac{I_{1}\left(K_{p}\right)}{I_{0}\left(K_{p}\right)}
$$

Here the total number of interacting pairs is denoted as $N_{B}$. The function $I_{1}(x)$ is the modified Bessel function $I_{1}(x)=\int_{0}^{2 \pi} \mathrm{d} \theta \cos \theta \mathrm{e}^{x \cos \theta}$.

We could carry out the calculation of the internal energy exactly owing to the gauge invariance of the Hamiltonian and the special condition $K=K_{p}$. The gauge invariance plays an important role in the gauge glass or random spin system because, for the gauge invariant quantities, the exact solution similar to the case of the internal energy can be calculated by using gauge transformation. The condition $K=K_{p}$ defines a line (the Nishimori line) in the phase diagram as in Figure 1. Although the line $K=K_{p}$ clearly runs across the phase boundary, the exact solution of the internal energy (5) is a simple function because the modified Bessel functions has no singularity. Thus, we may consider that the singular part of the internal energy vanishes on the Nishimori line. The same behavior of the internal energy as in the gauge glass appears in the Gaussian spin glass model and $\pm J$ spin glass model [11].

We can further evaluate some quantities using the spin wave approximation, which is a characteristic method in continuous spin systems, and obtain some information not available in Ising spin systems, which helps us to understand the significance of the Nishimori line. In particular, it is believed that the vortices created by the periodicity of eqn (1) cause the singularity relevant to the phase transition in the two-dimensional gauge glass. Accordingly, we expect that a new physical picture relevant to the Nishimori line may be found by using the characteristic method in the continuous spin system. This program is carried out in the next section. 

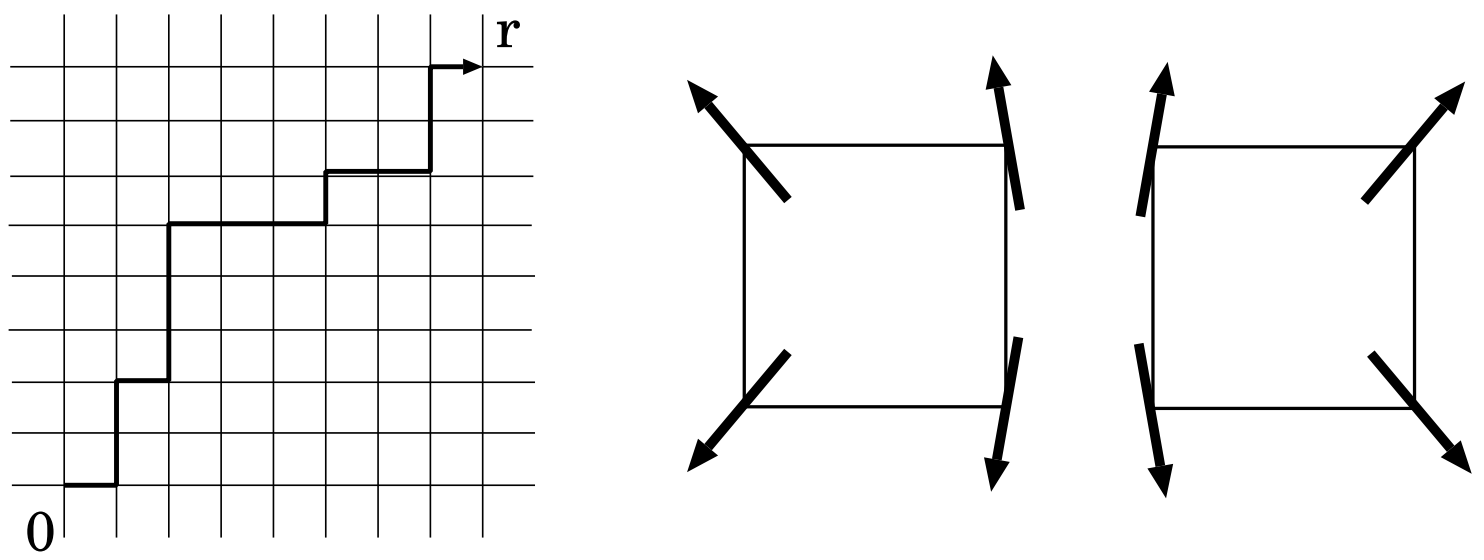

Fig. 2. Schematic diagram of the correlation function $C_{\mathrm{p}}(r)$ and chirality. Left figure shows a path from site 0 to site $r$. The right figure shows two states with different chiralities.

\section{Gauge invariant correlation functions}

\subsection{Definitions}

In order to investigate the physical significance of the result in the previous section, we introduce the gauge invariant correlation functions as

$$
\begin{aligned}
C_{\mathrm{p}} & =\left[\left\langle\operatorname{expi}\left(\theta_{0}-\theta_{r}-\sum_{k=1}^{r} A_{k-1, k}\right)\right\rangle\right] \\
C_{\mathrm{le}} & =\left[\left\langle\cos \left(\theta_{0}-\theta_{1}-A_{01}\right) \cos \left(\theta_{r}-\theta_{r+1}-A_{r, r+1}\right)\right\rangle\right] \\
C_{\mathrm{ch}} & =\left[\left\langle\sin \left(\theta_{0}-\theta_{1}-A_{01}\right) \sin \left(\theta_{r}-\theta_{r+1}-A_{r, r+1}\right)\right\rangle\right] .
\end{aligned}
$$

Equation (6) is the gauge-invariant correlation function which measures the correlation along a path from site 0 to site $r$ taking the phase twist of $A_{i j}$ into consideration. In Figure 2, we sketch a schematic picture of a path of correlation function $C_{\mathrm{p}}$. Equation (7) means the local energy correlation function. The local energy correlation function is also gauge invariant. Equation (8) represents the chirality correlation function. Chirality introduced by Villain [12] is an Ising-like degree of freedom. The chirality distinguishes the two states which are transformed to each other using the mirror transformation (Figure 2 ). The chirality correlation function is also gauge invariant. 


\subsection{Spin wave approximation}

Let us now consider the Hamiltonian introduced in eqn (1) in two dimensions. At sufficiently low temperatures, the effect of thermal fluctuations is small, so that the spins tend to align to each other with the twist $A_{i j}$ taken into account. Hence, we are able to expand the cosine term near $\left(\theta_{i}-\theta_{j}-A_{i j}\right)=0$,

$$
H \simeq \frac{J}{2} \sum_{\langle i, j\rangle}\left(\theta_{i}-\theta_{j}-A_{i j}\right)^{2}
$$

We note that this approximation neglects periodicity of cosine in eqn (1). It is generally believed that, if we want to describe the phase transition in twodimensional gauge glass, we must consider the contribution of periodicity or vortices [7]. To ensure the validity of the expansion of eqn (9), we must assume that the phase twist $A_{i j}$ is sufficiently small, so that eqn (2) is expressed as

$$
P\left(A_{i j}\right) \propto \mathrm{e}^{-K_{p} A_{i j}^{2} / 2}
$$

The constant $K_{p}^{-1}$ is equal to the variance of the Gaussian distribution $\left[A_{i j}^{2}\right] \equiv$ $\sigma$. In the same way as in the spin wave approximation, the distribution of eqn (10) neglects periodicity of $A_{i j}$. This approximation of the distribution of $A_{i j}$ is expected to be sufficient to describe correlations at low temperature.

Using the spin wave approximation of eqns (9) and (10), we can calculate the gauge invariant correlation functions as

$$
\begin{aligned}
C_{\mathrm{p}} & =\exp \left\{\frac{1}{2}\left(\frac{1}{K_{p}}-\frac{1}{K}\right) G_{0 r}-\frac{\sigma r}{2}\right\} \\
C_{\mathrm{le}} & =\frac{1}{2} \exp \left\{\left(\frac{1}{K_{p}}-\frac{1}{K}\right) G_{0 r}^{(-)}-\sigma\right\}+\frac{1}{2} \exp \left\{\left(\frac{1}{K_{p}}-\frac{1}{K}\right) G_{0 r}^{(+)}-\sigma\right\}(12) \\
C_{\mathrm{ch}} & =\frac{1}{2} \exp \left\{\left(\frac{1}{K_{p}}-\frac{1}{K}\right) G_{0 r}^{(-)}-\sigma\right\}-\frac{1}{2} \exp \left\{\left(\frac{1}{K_{p}}-\frac{1}{K}\right) G_{0 r}^{(+)}-\sigma\right\}(, 1)
\end{aligned}
$$

where $G_{0 r}$ and $G_{0 r}^{( \pm)}$are the lattice Green functions,

$$
\begin{aligned}
G_{0 r} & =\frac{1}{N} \sum_{\boldsymbol{k}} \frac{1-\cos \boldsymbol{k} \cdot \boldsymbol{r}}{4-2 \cos k_{x}-2 \cos k_{y}} \\
G_{0 r}^{( \pm)} & =\frac{1}{N} \sum_{\boldsymbol{k}} \frac{\left(1-\cos k_{1}\right)(1 \pm \cos \boldsymbol{k} \cdot \boldsymbol{r})}{2-\cos k_{1}-\cos k_{2}} .
\end{aligned}
$$


Now, applying the special condition $K=K_{p}$, we can obtain these correlation functions as follows,

$$
C_{\mathrm{p}}=\mathrm{e}^{-K_{p}^{-1} r / 2}, \quad C_{\mathrm{le}}=\mathrm{e}^{K_{p}^{-1}}, C_{\mathrm{ch}}=0 .
$$

Surprisingly, the chirality correlation function vanishes under the condition $K=K_{p}$. The chirality degrees of freedom behave perfectly independently from place to place.

\subsection{Gauge transformation}

From comparison of the results of the spin wave approximation with the exact results, we can investigate the difference between the model with periodicity and the model without periodicity. In this section, we calculate the exact solutions of $C_{\mathrm{p}}, C_{\mathrm{le}}$ and $C_{\mathrm{ch}}$ for models with periodicity using gauge transformation under the special condition $K=K_{p}$. After the derivations of the exact solutions of correlation functions, we study the asymptotic forms of the exact solutions at low temperatures and compare them with the solutions in the previous section using the spin wave approximation.

The correlation functions which we introduced in Section 3.1 are invariant under the gauge transformation, and therefore the same calculation method as for the internal energy (5) in Section 2 can be applied to the calculation of the correlation functions. As a consequence, we obtain the following expression under the special condition $K=K_{p}$,

$$
C_{\mathrm{p}}=\left(\frac{I_{1}(K)}{I_{0}(K)}\right)^{r}, \quad C_{\mathrm{le}}=\left(\frac{I_{1}(K)}{I_{0}(K)}\right)^{2}, \quad C_{\mathrm{ch}}=0
$$

When $K=K_{p}$, the chirality correlation vanishes exactly and this solution is consistent with the result of spin wave approximation (16). At low temperature, the asymptotic behaviors of $C_{\mathrm{p}}$ and $C_{\mathrm{le}}$ in eqn (17) are written as follows,

$$
C_{\mathrm{p}} \sim \mathrm{e}^{-K_{p}^{-1} r / 2}, \quad C_{\mathrm{le}} \sim \mathrm{e}^{-K_{p}^{-1}}
$$

Equation (18) is also consistent with the solution of spin wave approximation expressed in eqn (16). This comparison suggests that under the special condition $K=K_{p}$, the system forms a characteristic structure which is not influenced by the vortices. The analysis of the Villain model in the following

reinforces this picture. 


\subsection{Villain model}

The Hamiltonian of the spin wave approximation (9) neglects periodicity included in the cosine term (1). If we need to describe the system at low temperatures more accurately, it should be necessary to consider periodicity in the Hamiltonian. Accordingly, we consider the Villain model which adds the periodicity to the spin wave Hamiltonian

$$
Z_{V}=\int_{0}^{2 \pi} \prod_{i} \frac{\mathrm{d} \theta_{i}}{2 \pi} \sum_{\left\{m_{i j}=-\infty\right\}}^{\infty} \mathrm{e}^{-\frac{K}{2} \sum_{\langle i, j\rangle}\left(\theta_{i}-\theta_{j}-A_{i j}-2 m_{i j} \pi\right)^{2}}
$$

where $m_{i j}$ is an integer between $-\infty$ and $\infty$.

The random phase shift (10) also neglects the periodicity of modulo $2 \pi$. Therefore we also add $2 m_{i j} \pi$ into the probability distribution of $A_{i j}(10)$. The configurational average is described as

$$
[\cdots]=\int_{0}^{2 \pi} \prod_{\langle i, j\rangle} \frac{\mathrm{d} A_{i j}}{\sqrt{2 \pi K_{p}^{-1}}}(\cdots) \sum_{\left\{m_{i j}=-\infty\right\}}^{\infty} \mathrm{e}^{-\frac{K_{p}}{2}\left(A_{i j}-2 m_{i j} \pi\right)^{2}}
$$

From eqns (19) and (20) and the gauge transformation, we can calculate the correlation functions discussed in the previous section. We first consider the gauge-invariant correlation function $C_{\mathrm{p}}$. Applying the gauge transformation and the condition $K=K_{p}$, we obtain the following form,

$$
\begin{aligned}
C_{\mathrm{p}}= & {\left[\left\langle\operatorname{expi}\left(\theta_{0}-\theta_{r}-\sum_{k=1}^{r} A_{k-1 k}\right)\right\rangle\right] } \\
= & \int_{0}^{2 \pi} \prod_{\langle i, j\rangle} \frac{\mathrm{d} A_{i j}}{\sqrt{2 \pi K_{p}^{-1}}} \sum_{\left\{m_{i j}=-\infty\right\}}^{\infty} \\
& \times \int_{0}^{2 \pi} \prod_{i} \frac{\mathrm{d} \theta_{i}}{2 \pi} \mathrm{e}^{\mathrm{i}\left(\theta_{0}-\theta_{r}-\sum_{k} A_{k-1 k}\right)} \mathrm{e}^{-\frac{K_{p}}{2} \sum_{\langle i, j\rangle}\left(\theta_{i}-\theta_{j}-A_{i j}-2 m_{i j} \pi\right)^{2}} .
\end{aligned}
$$

To integrate over $A_{i j}$, we use the following relation,

$$
\begin{aligned}
\int_{0}^{2 \pi} \frac{\mathrm{d} A_{i j}}{\sqrt{2 \pi K_{p}^{-1}}} \sum_{m_{i j}=-\infty}^{\infty} & \mathrm{e}^{-\frac{K_{p}}{2}\left(\theta_{i}-\theta_{j}-A_{i j}-2 m_{i j} \pi\right)^{2}} \\
& =\int_{-\infty}^{\infty} \frac{\mathrm{d} A_{i j}}{\sqrt{2 \pi K_{p}^{-1}}} \mathrm{e}^{-\frac{K_{p}}{2}\left(\theta_{i}-\theta_{j}-A_{i j}\right)^{2}}
\end{aligned}
$$


Substituting eqn (22) into eqn (21), we obtain the gauge-invariant correlation function as

$$
C_{\mathrm{p}}=\mathrm{e}^{-K_{p}^{-1} r / 2}
$$

where we have used the relation $K=K_{p}$. The result of this calculation agrees with the one of the spin wave approximation (16) and the asymptotic form of the exact solution (18) under the condition $K=K_{p}$. The local energy correlation function and the chirality correlation function are derived by the same calculation as in the gauge invariant correlation. These results are written by simple formulas as

$$
C_{\mathrm{le}}=\mathrm{e}^{-K_{p}^{-1}}, C_{\mathrm{ch}}=0
$$

Those two correlation functions also agree with the results of the spin wave approximation (16) and asymptotic exact solution (18) under the condition $K=K_{p}$. We may conclude from these results as follows. The spin wave approximation neglects periodicity, and the results of the spin wave approximation do not include the effects of vortices. The results for the Villain model in contrast include the effects of vortices. Accordingly, the agreement of the results of spin wave approximation with those of the Villain model suggests the absence of the effects of vortices on the correlation functions we calculated under the condition $K=K_{p}$. Vortices are perfectly irrelevant to the gauge invariant correlation functions.

\section{Distribution functions}

Irrelevance of vortices in the gauge invariant correlation functions on the Nishimori line can be verified by using a distribution function with gauge invariance. This gauge invariant distribution function measures the distribution of the random phase twist between site $i$ and $j$. The definition is

$$
P(x)=\left[\left\langle\delta\left(x-\left(\theta_{i}-\theta_{j}-A_{i j}\right)\right)\right\rangle\right]
$$

Using the spin wave approximation and the Villain model, we obtain the comparable results as in the gauge invariant correlation functions when $K=$ $K_{p}$. The results using the spin wave approximation and the Villain model are, respectively, 


$$
\begin{aligned}
P_{\mathrm{sw}}(x) & =\sqrt{\frac{K_{p}}{2 \pi}} \exp \left(-\frac{K_{p}}{2} x^{2}\right) \\
P_{\text {Villain }}(x) & =\sqrt{\frac{K_{p}}{2 \pi}} \exp \left(-\frac{K_{p}}{2} x^{2}\right),
\end{aligned}
$$

the latter being the exact solution. These two expressions are completely identical.

The distribution function of two bonds can also be calculated by the spin wave approximation and the Villain model. We first show the definition of the two-bond distribution function

$$
P(x, y)=\left[\left\langle\delta\left(x-\left(\theta_{k}-\theta_{l}-A_{k l}\right)\right) \cdot \delta\left(y-\left(\theta_{m}-\theta_{n}-A_{m n}\right)\right)\right\rangle\right] .
$$

Equation (28) is calculated by using the spin wave approximation and the Villain model for $K=K_{p}$ as, again the latter being the exact solution,

$$
\begin{aligned}
P_{\mathrm{sw}}(x, y) & =\frac{K_{p}}{2 \pi} \exp \left(-\frac{K_{p}}{2}\left(x^{2}+y^{2}\right)\right) \\
P_{\text {Villain }}(x, y) & =\frac{K_{p}}{2 \pi} \exp \left(-\frac{K_{p}}{2}\left(x^{2}+y^{2}\right)\right) .
\end{aligned}
$$

Similarly to eqns (26) and (27), these results agree with each other. We therefore see immediately that vortices have no effects on the distribution function (28). It also follows that two-bond variables behave independently because eqns $(29) /(30)$ are just products of eqns $(26) /(27)$.

It is instructive to consider another two-bond extension,

$$
P^{\prime}(x, y)=\left[\left\langle\delta\left(x-\left(\theta_{k}-\theta_{l}-A_{k l}\right)\right)\right\rangle \cdot\left\langle\delta\left(y-\left(\theta_{m}-\theta_{n}-A_{m n}\right)\right)\right\rangle\right]
$$

Although eqn (31) is gauge invariant, we can not calculate the exact solution even if we apply gauge transformation to eqn (31). Nevertheless, we can obtain the result by the spin wave approximation as

$$
P_{\mathrm{sw}}^{\prime}(x, y)=\frac{K_{p}}{2 \pi \sqrt{1-A^{2}}} \exp \left(-\frac{K_{p}}{2\left(1-A^{2}\right)}\left(x^{2}+y^{2}-2 A x y\right)\right)
$$

where $A \equiv G_{k n}-G_{l m}-G_{k m}+G_{l n}$. In contrast to eqn (28), the distribution function (31) can not be written as the products of eqn (26) under the condition $K=K_{p}$. Bond variables are correlated in this sense. Inequivalence of eqns (28) and (31) is a highly non-trivial fact. If we measure the two-bond correlation within a given system as in eqn (28), the result becomes uncorrelated: $P(x, y)=P(x) P(y)$. If we, on the other hand, look at the correlation 
of the thermal averages of single-bond distribution function at two different locations as in eqn (31), these are correlated: $P^{\prime}(x, y) \neq P(x) P(y)$. Physical significance of this counter-intuitive results needs further clarification.

\section{$5 \quad$ Summary and discussions}

In this paper, we have investigated the properties of the gauge glass on the Nishimori line through the gauge invariant quantities. Firstly we calculated the gauge invariant correlation functions using the spin wave approximation, gauge transformation and the Villain model. From these calculations, we found that the results of the spin wave approximation are identical to the results by the method taking periodicity into account. Thus, we expect that the periodicity or vortices has no influence on the system (gauge invariant quantities to be precise) under the condition $K=K_{p}$. The effect of vortices can be neglected on the Nishimori line.

Next, we calculated the gauge invariant distribution functions using the spin wave approximation and the Villain model. We found the same results as for the gauge invariant correlation functions. Moreover, we showed that the distribution function (28) is independently distributed at each bond. An variant of eqn (28) was also calculated by using the spin wave approximation.

We may conclude from these results as follows. In the two-dimensional gauge glass, the effect of vortices plays an important role because the phase transition of the model is described by vortex-pair unbinding. Accordingly, the spin wave approximation which neglects the effect of vortices is considered in general not to be able to describe the singularity related to the phase transition. However, our analysis based on the gauge invariant quantities predicts that under the condition $K=K_{p}$, the singularity related to the phase transition vanishes for gauge invariant correlation functions even if we consider the effect of vortices in the system. Thus, the renormalization group arguments [7][13], which analyze the effects of vortices asymptotically, should be reconsidered seriously under the present perspective. Further investigation of the property of the Nishimori line is necessary.

\section{References}

[1] M. Rubinstein, B. Shraiman and D. R. Nelson, Phys. Rev. B 27, 1800 (1983).

[2] E. Granato and J. M. Kosterlitz, Phys. Rev. B 33, 6553 (1986).

[3] W. Y. Shih, C. Ebner and D. Stroud, Phys. Rev. B 30, 134 (1984).

[4] T. Nattermann, S. Scheidl, S. E. Korshunov and M. S. Li, J. Phys. (France) 5, 565 (1995). 
[5] S. Sheidl, Phys. Rev. B 55, 457 (1997).

[6] J. Maucourt and D. R. Grempel, Phys. Rev. B 56, 2572 (1997).

[7] D. Carpentier and P. Doussal, Nucl. Phys. B 588, 565 (1993).

[8] C. Mudry and X-G. Wen, Nucl. Phys. B 549, 613 (1999).

[9] H. Nishimori, Prog. Theor. Phys. 66, 1169 (1981).

[10] Y. Ozeki and H. Nishimori, J. Phys. A 26, 3399 (1993).

[11] H. Nishimori, "Statistical Physics of Spin Glasses and Information Processing: An Introduction" Oxford University Press, (2001).

[12] J. Villain, J. Phys. C 10, 4793 (1977).

[13] L-H. Tang, Phys. Rev. B 54, 3350-3366 (1996). 